\title{
Commentaries
}

\section{Determination of the molecular basis of Marfan syndrome: a growth industry}

\author{
Peter H. Byers
}

Departments of Pathology and Medicine, University of Washington, Seattle, Washington, USA.

\begin{abstract}
Although it has been known for more than a decade that Marfan syndrome - a dominantly inherited connective tissue disorder characterized by tall stature, arachnodactyly, lens subluxation, and a high risk of aortic aneurysm and dissection - results from mutations in the FBN1 gene, which encodes fibrillin-1, the precise mechanism by which the pleiotropic phenotype is produced has been unclear. A report in this issue now proposes that loss of fibrillin-1 protein by any of several mechanisms and the subsequent effect on the pool of TGF- $\beta$ may be more relevant in the development of Marfan syndrome than mechanisms previously proposed in a dominant-negative disease model (see the related article beginning on page 172). The model proposed in this issue demonstrates several strategies for clinical intervention.
\end{abstract}

The identification in 1991 of the FBN1 gene, which encodes the fibrillin-1 protein, as the locus for mutations that result in Marfan syndrome (1) was the culmination of four complementary lines of investigation, including (a) demonstration of fibrillin- 1 in tissues affected in Marfan syndrome, such as lens zonular fibrils $(2,3)$; (b) genetic linkage of the Marfan phenotype to the long arm of chromosome 15 (4); (c) demonstration that the FBN1 gene is located on $15 \mathrm{q}(5)$; and (d) recognition that cultured fibroblasts from people with Marfan syndrome often had defects in the synthesis, secretion, or assembly of fibrillin (6). Since then more than 600 mutations in the FBN1 gene have been identified (7), and the list of known phenotypic effects resulting from these mutations has been extended. The clinical phenotypes that can result from mutations in the gene include, in addition to Marfan syndrome, isolated ectopia lentis, a rare autosomal dominant form of Weill-Marchesani syndrome (8), kyphoscoliosis (9), aneurysm of the ascending aorta with only minor skeletal anomalies (10), skeletal and ocular involvement (10), and Spritzen-Goldberg syndrome (Marfanoid features with craniosynostosis) (11). In mice, duplication of a central portion of the Fbn1 gene (exons 17-40) results in a tight skin phenotype (12).

Nonstandard abbreviations used: latency-associated protein (LAP); latent TGF- $\beta$-binding protein (LTBP).

Conflict of interest: The author has declared that no conflict of interest exists.

Citation for this article:

J. Clin. Invest. 114:161-163 (2004).

doi:10.1172/JCI200422399.
At the time FBN1 mutations were identified it appeared that the game was up in terms of understanding the molecular pathogenesis of Marfan syndrome. Fibrillin-1 was known to be a major component of the extracellular matrix structures known as microfibrils upon which elastin appeared to be deposited. Therefore, the loss of aortic integrity associated with the syndrome was reasoned to be the consequence of altered microfibril structural integrity. After all, fibrillin-1 molecules aggregated in an orderly fashion to form fibrils that contained many molecules. The presence of a population of abnormal molecules could, it was thought, alter the entire fibril structure (a dominant-negative effect in which, because many molecules interact, the presence of some abnormal ones is amplified), given the stringent structural requirements to produce intact fibrils. Expression of mutant FBN1 cDNA in normal human or mouse cells replicates the effects seen in cells from individuals with a single mutant allele and a normal allele, suggesting that the dominant-negative effect explained the phenotype (13). Some features of Marfan syndrome were, however, difficult to reconcile with this model. These features include long bone and rib overgrowth, and alterations in muscle growth and fat metabolism - effects not often considered to be consequences of defects in matrix protein structure or synthesis. Observations such as these prompted a reconsideration of whether a dominant-negative model for the molecular pathogenesis of Marfan syndrome was, in fact, accurate.
In this issue of the JCI, Judge and colleagues test the hypothesis that haploinsufficiency is the major effect rather than the dominantnegative effect expected with multimeric proteins (14). They used two lines of approach. First, they found that overexpression in mice of a Marfan syndrome-associated mutant form of human fibrillin-1 (C1663R) does not result in an observable Marfan syndromerelated phenotype, even though the abnormal human molecule appears to be incorporated into microfibril structures along with the endogenous murine fibrillin- 1 molecules with which the human molecules interact. Second, they observed that the human FBN1 gene appears to rescue the Marfan syndrome-like vascular phenotype observed in mice heterozygous for the $\mathrm{C} 1039 \mathrm{G}$ mutation in murine Fbn1. If it is indeed the presence of the abnormal molecules that results in the Marfan syndrome phenotype, these results are unexpected. The data certainly suggest that in individuals with Marfan syndrome the loss of products of one allele is an important component of the phenotype. Judge and colleagues cite examples in which deletion of one FBN1 allele in humans produces a mild Marfan phenotype. The experiments they performed in mice do not exclude the possibility that the human gene - even a mutant copy of the gene, which also provides some amelioration of the murine phenotype - could potentially play a different and more advantageous role in the mouse. Future experiments in which increased numbers of copies of the normal murine gene are introduced on the background of 1 or 2 mutant Fbn1 alleles could provide a more definitive answer to the question of what is the major driving force behind the phenotype. Even so, the studies reported by Judge and colleagues serve to emphasize that we do not yet fully understand the molecular pathogenesis of Marfan syndrome and further suggest that something very interesting is afoot.

\section{Fibrillin structure and function}

There are three identified human fibrillin genes: FBN1, FBN2, and FBN3. They share a pattern of structural motifs that includes 


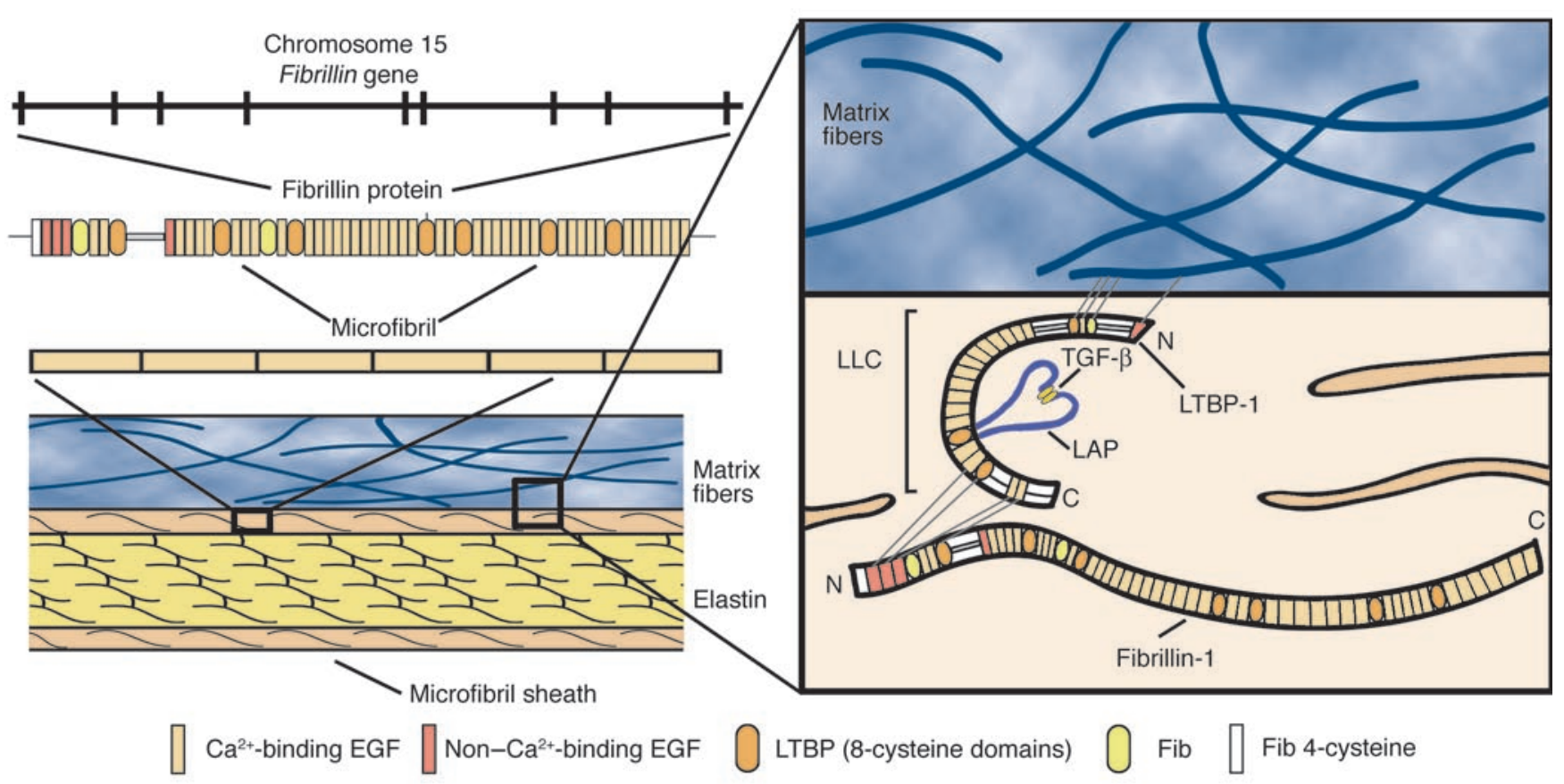

Figure 1

Fibrillin-1 is translated from an mRNA encoded by the FBN1 gene on chromosome 15. The protein is processed and secreted and then forms microfibrils in the matrix in association with other extracellular molecules. Microfibrils may function alone or form the basis of elastic fibers following deposition of elastin. Among molecules that are associated with fibrillin-1 is LTBP-1, a binding protein for the latent form of TGF- $\beta$. TGF- $\beta$ is expressed as a precursor, which is cleaved to make the active form, but the two peptides remain associated and are then bound by a separate protein, LTBP-1. This complex, known as a large latent complex (LLC) is bound to elements of the extracellular matrix, including fibrillin-1 and other fibrils, and TGF- $\beta$ is released in response to environmental and molecular signals. Although the traditional model for Marfan syndrome suggests that it is the alterations in the structure of fibrillin-1 that are important, the article by Judge and colleagues in this issue of the $\mathrm{JCl}(14)$ suggests that expression of only half the normal amount of wild-type fibrillin-1 causes global impairment in the deposition of microfibrils. This could have dramatic effects on tissue structure and function. Figure adapted with permission from Nature Genetics (26).

more than 40 calcium-binding EGF-precursor-like units arranged in similar sized blocks separated by modules known as 8 -cysteine or TGF- $\beta$-binding domains (Figure 1). The EGF-precursor motifs are seen in many proteins, but the 8-cysteine motifs are confined to the family of proteins that include latent TGF- $\beta$-binding proteins (LTBPs), which are smaller than but very similar in design to fibrillins, and fibrillins. Mutations in the FBN2 gene give rise to contractural arachnodactyly (15), while FBN3 is a candidate gene for recessively inherited Weill-Marchesani syndrome and is disrupted in the mouse genome by an ancient murinespecific chromosomal translocation (16). The LTBPs play an important role in providing a safe harbor for TGF- $\beta$ species prior to their activation and release by perturbation of elements of the extracellular matrix (17) (Figure 1). The sequence and structural similarities of the LTBPs and fibrillin suggest that at least fibrillin-1 could either provide a binding site for latent TGF- $\beta$ or interact with the LTBPs and provide a monitor of the integrity of the ECM (18). Fibrillin-1 itself does not appear to bind TGF- $\beta$, even though the 8-cysteine motifs are very similar to those in the LTBPs (19). An N-terminal domain of fibrillin-1 does, however, bind to LTBP-1 through a C-terminal region of the latter (Figure 1). In this configuration, alterations in the matrix are sufficient to release TGF- $\beta$ from its latency-associated protein (LAP), which is bound to LTBP-1 so that it can signal nearby cells (20).

\section{Fibrillin: more than a structural protein}

The idea that fibrillin-1 is more than just a structural protein and could participate in TGF- $\beta$-related signaling got its biggest boost from two findings. First, the large fibrillin-1 molecule produced in the tight skin mice appeared to bind substantially more TGF- $\beta$, presumably in the LAP-LTBP-1 bound form, than the normal fibrillin-1 molecules (21). Second, studies of lung development in mice that had low fibrillin-1-producing engineered mutations of the Fbn 1 gene showed that those mice had markedly elevated levels of TGF- $\beta$ in developing lungs, had fewer but larger alveoli, and developed emphysematous changes in the lungs as they aged. (22). The alteration in alveolar septation was corrected by treatment of the animals in utero with antibodies to TGF- $\beta$, which strongly implicates this molecule as a major factor in the abnormal pulmonary development seen in Marfan syndrome. Thus, the amount of fibrillin- 1 in the matrix may be one determinant of the reservoir for TGF- $\beta$, and either an increase or decrease in the number of fibrillin-1 molecules might have phenotypic effects. This effect could be further modulated by the presence of high- or low-expressing variants of $F B N 1$, which are known to play a role in variable expression of the Marfan phenotype (23). It seems plausible that, in addition, alteration of the structure of fibrillin-1 might contribute to the phenotype when mutations diminish the ability of fibrillin-1 to bind the LTBPs and thus release more TGF- $\beta$ into the matrix (20).

It is the mantra of human molecular genetics that understanding the molecular basis of human genetic disease will lead to specific and effective treatment. To date this 
hope has often been more hype than realization. The story of Marfan syndrome and mutations in the FBN1 gene illustrates that identifying a mutation is only the first step in understanding the molecular pathophysiology of the disorder and that the pathway to treatment may be both complex and full of insights into unexpected areas of biology $(24,25)$. Perhaps in a short time we may see the use of TGF- $\beta$ blockade in preference to $\beta$-(adrenergic) blockade as a more effective treatment of many aspects of Marfan syndrome - and treatment is the ultimate goal of understanding this complex phenotype.

Address correspondence to: Peter H. Byers, Department of Pathology, Box 357470, University of Washington, Seattle, Washington 98195-7470, USA. Phone: (206) 543-4206; Fax: (206) 616-1899; E-mail: pbyers@u.washington.edu.

1. Dietz, H.C., et al. 1991. Marfan syndrome caused by a recurrent de novo missense mutation in the fibrillin gene. Nature. 352:337-339.

2. Sakai, L.Y., Keene, D.R., and Engvall, E. 1986. Fibrillin, a new $350-\mathrm{kD}$ glycoprotein, is a component of extracellular microfibrils. J. Cell Biol. 103:2499-2509.

3. Hollister, D.W., Godfrey, M., Sakai, L.Y., and Pyeritz, R.E. 1990. Immunohistologic abnormalities of the microfibrillar-fiber system in the Marfan syndrome. N. Engl. J. Med. 323:152-159.

4. Lee, B., et al. 1991. Linkage of Marfan syndrome and a phenotypically related disorder to two different fibrillin genes. Nature. 352:330-334.
5. Magenis, R.E., Maslen, C.L., Smith, L., Allen, L., and Sakai, L.Y. 1991. Localization of the fibrillin (FBN) gene to chromosome 15, band q21.1. Genomics. 11:346-351.

6. Milewicz, D.M., Pyeritz, R.E., Crawford, E.S., and Byers, P.H. 1992. Marfan syndrome: defective synthesis, secretion, and extracellular matrix formation of fibrillin by cultured dermal fibroblasts. J. Clin. Invest. 89:79-86.

7. The FBN1 mutations database. http://www.umd. be:2030/.

8. Faivre, L., et al. 2003. In frame fibrillin-1 gene deletion in autosomal dominant Weill-Marchesani syndrome. J. Med. Genet. 40:34-36.

9. Ades, L.C., et al. 2002. Segregation of a novel FBN1 gene mutation, G1796E, with kyphoscoliosis and radiographic evidence of vertebral dysplasia in three generations. Am. J. Med. Genet. 109:261-270.

10. Katzke, S., et al. 2002. TGGE screening of the entire FBN1 coding sequence in 126 individuals with marfan syndrome and related fibrillinopathies. Hum. Mutat. 20:197-208.

11. Sood, S., Eldadah, Z.A., Krause, W.L., McIntosh, I., and Dietz, H.C. 1996. Mutation in fibrillin-1 and the Marfanoid-craniosynostosis (Shprintzen-Goldberg) syndrome. Nat. Genet. 12:209-211.

12. Bona, C.A., et al. 1997. Structure of the mutant fibrillin-1 gene in the tight skin (TSK) mouse. DNA Res. 4:267-271.

13. Eldadah, Z.A., Brenn, T., Furthmayr, H., and Dietz, H.C. 1995. Expression of a mutant human fibrillin allele upon a normal human or murine genetic background recapitulates a Marfan cellular phenotype. J. Clin. Invest. 95:874-880.

14. Judge, D.P., et al. 2004. Evidence for a critical contribution of haploinsufficiency in the complex pathogenesis of Marfan syndrome. J. Clin. Invest. 114:172-181. doi:10.1172/JCI200420641.

15. Putnam, E.A., Zhang, H., Ramirez, F., and Milewicz, D.M. 1995. Fibrillin-2 (FBN2) mutations result in the Marfan-like disorder, congenital contractural arachnodactyly. Nat. Genet. 11:456-458.
16. Corson, G.M., Charbonneau, N.L., Keene, D.R., and Sakai, L.Y. 2004. Differential expression of fibrillin-3 adds to microfibril variety in human and avian, but not rodent, connective tissues. Genomics. 83:461-472.

17. Annes, J.P., Munger, J.S., and Rifkin, D.B. 2003. Making sense of latent TGFbeta activation. J. Cell. Sci. 116:217-224.

18. Nakajima, Y., Miyazono, K., and Nakamura, H. 1999. Immunolocalization of latent transforming growth factor-beta binding protein-1 (LTBP1) during mouse development: possible roles in epithelial and mesenchymal cytodifferentiation. Cell Tissue Res. 295:257-267.

19. Saharinen, J., and Keski-Oja, J. 2000. Specific sequence motif of 8-Cys repeats of TGF-beta binding proteins, LTBPs, creates a hydrophobic interaction surface for binding of small latent TGF-beta. Mol. Biol. Cell. 11:2691-2704.

20. Kaartinen, V., and Warburton, D. 2003. Fibrillin controls TGF-beta activation. Nat. Genet. 33:331-332.

21. Saito, S., et al. 1999. Characterization of mutated protein encoded by partially duplicated fibrillin-1 gene in tight skin (TSK) mice. Mol. Immunol. 36:169-176.

22. Neptune, E.R., et al. 2003. Dysregulation of TGF-beta activation contributes to pathogenesis in Marfan syndrome. Nat. Genet. 33:407-411.

23. Hutchinson, S., et al. 2003. Allelic variation in normal human FBN1 expression in a family with Marfan syndrome: a potential modifier of phenotype? Hum. Mol. Genet. 12:2269-2276.

24. Aoyama, T., Tynan, K., Dietz, H.C., Francke, U., and Furthmayr, H. 1993. Missense mutations impair intracellular processing of fibrillin and microfibril assembly in Marfan syndrome. Hum. Mol. Genet. 2:2135-2140

25. Frischmeyer, P.A., and Dietz, H.C. 1999. Nonsensemediated mRNA decay in health and disease. Hum. Mol. Genet. 8:1893-1900.

26. Kaartinen, V., and Warburton, D. 2003. Fibrillin controls TGF- $\beta$ activation. Nat. Genet. 33:331-332.

\title{
Unbuckling lipodystrophy from insulin resistance and hypertension
}

\author{
Robert A. Hegele ${ }^{1}$ and Todd Leff ${ }^{2}$
}

\begin{abstract}
${ }^{1}$ Robarts Research Institute, London, Ontario, Canada. ${ }^{2}$ Department of Pathology, Wayne State University School of Medicine, Detroit, Michigan, USA.
\end{abstract}

\begin{abstract}
Lipodystrophy and insulin resistance are the core features of human PPAR $\gamma$ deficiency states. Metabolic complications in PPAR $\gamma$ deficiency, such as hypertension, have been considered to be secondary to insulin resistance. However, a new mouse model that expresses the analog of a human PPARG mutation displays minimal lipodystrophy and insulin resistance but rather severe hypertension (see the related article beginning on page 240). Furthermore, the mutant protein appears to directly modulate the renin-angiotensin system in adipose tissue, providing evidence of the pleiotropic effects of PPAR $\gamma$.
\end{abstract}

Nonstandard abbreviations used: angiotensin II (ATII); familial partial lipodystrophy type 3 (FPLD3); metabolic syndrome (MetS); renin-angiotensin system (RAS); type 2 diabetes mellitus (T2DM).

Conflict of interest: The authors have declared that no conflict of interest exists.

Citation for this article:

J. Clin. Invest. 114:163-165 (2004).

doi:10.1172/JCI200422382.
The commonly occurring metabolic syndrome (MetS) is considered to result from complex gene-environment interactions and has been associated with future onset of type 2 diabetes mellitus (T2DM) (1) and both all-cause and cardiovascular mortality (2). MetS is defined clinically according to deviation from threshold values for three or more of five quantitative traits; namely, waist circumference, blood pressure, and plasma concentrations of glucose, highdensity lipoprotein cholesterol, and triglyceride (3). Insulin resistance has long been considered the core biochemical defect linking these metabolic disturbances, which are strongly correlated among and within patients, suggesting the existence of common underlying molecular mechanisms. No molecule is more central to the metabolic and vascular pathways of MetS than PPAR $\gamma$ (4). The effect of altered activity of the PPAR $\gamma$ receptor on whole-body insulin sensitivity has been appreciated for years. For instance, in both mice and humans, activating PPAR $\gamma$ ligands has ben- 